\title{
Expression of Cellulosome Components and Type IV Pili within the Extracellular Proteome of Ruminococcus flavefaciens 007
}

\author{
Maša Vodovnik, ${ }^{1,2}$, Sylvia H. Duncan ${ }^{2}$, Martin D. Reid ${ }^{2}$, Louise Cantlay ${ }^{2}$, Keith Turner ${ }^{3}$, Julian Parkhill ${ }^{3}$, \\ Raphael Lamed ${ }^{5}$, Carl J. Yeoman ${ }^{6}$, Margret E. Berg Miller ${ }^{6}$, Bryan A. White ${ }^{6,7}$, Edward A. Bayer ${ }^{4}$, \\ Romana Marinšek-Logar ${ }^{1}$, Harry J. Flint ${ }^{2 *}$
}

1 Chair for Microbiology and Microbial Biotechnology, Biotechnical Faculty, University of Ljubljana, Ljubljana, Slovenia, 2 Rowett Institute of Nutrition and Health, University of Aberdeen, Aberdeen, United Kingdom, $\mathbf{3}$ Wellcome Trust Sanger Institute, Cambridge, United Kingdom, 4 Department of Biological Chemistry, The Weizmann Institute of Science, Rehovot, Israel, $\mathbf{5}$ Department of Molecular Microbiology and Biotechnology, Tel Aviv University, Ramat Aviv, Israel, $\mathbf{6}$ Department of Animal Sciences, University of Illinois at Urbana-Champaign, Urbana, Illinois, United States of America, 7 Institute for Genomic Biology, University of Illinois at UrbanaChampaign, Urbana, Illinois, United States of America

\begin{abstract}
Background: Ruminococcus flavefaciens is an important fibre-degrading bacterium found in the mammalian gut. Cellulolytic strains from the bovine rumen have been shown to produce complex cellulosome structures that are associated with the cell surface. R. flavefaciens 007 is a highly cellulolytic strain whose ability to degrade dewaxed cotton, but not Avicel cellulose, was lost following initial isolation in the variant $007 \mathrm{~S}$. The ability was recovered after serial subculture to give the cotton-degrading strain $007 \mathrm{C}$. This has allowed us to investigate the factors required for degradation of this particularly recalcitrant form of cellulose.

Methodology/Principal Findings: The major proteins associated with the bacterial cell surface and with the culture supernatant were analyzed for $R$. flavefaciens $007 \mathrm{~S}$ and $007 \mathrm{C}$ grown with cellobiose, xylan or Avicel cellulose as energy sources. Identification of the proteins was enabled by a draft genome sequence obtained for 007C. Among supernatant proteins a cellulosomal GH48 hydrolase, a rubrerthyrin-like protein and a protein with type IV pili N-terminal domain were the most strongly up-regulated in $007 \mathrm{C}$ cultures grown on Avicel compared with cellobiose. Strain 007S also showed substrate-related changes, but supernatant expression of the Pil protein and rubrerythrin in particular were markedly lower in $007 \mathrm{~S}$ than in $007 \mathrm{C}$ during growth on Avicel.

Conclusions/Significance: This study provides new information on the extracellular proteome of $R$. flavefaciens and its regulation in response to different growth substrates. Furthermore it suggests that the cotton cellulose non-degrading strain (007S) has altered regulation of multiple proteins that may be required for breakdown of cotton cellulose. One of these, the type IV pilus was previously shown to play a role in adhesion to cellulose in $R$. albus, and a related pilin protein was identified here for the first time as a major extracellular protein in $R$. flavefaciens.
\end{abstract}

Citation: Vodovnik M, Duncan SH, Reid MD, Cantlay L, Turner K, et al. (2013) Expression of Cellulosome Components and Type IV Pili within the Extracellular Proteome of Ruminococcus flavefaciens 007. PLoS ONE 8(6): e65333. doi:10.1371/journal.pone.0065333

Editor: Marie-Joelle Virolle, University Paris South, France

Received November 12, 2012; Accepted April 24, 2013; Published June 4, 2013

Copyright: (c) 2013 Vodovnik et al. This is an open-access article distributed under the terms of the Creative Commons Attribution License, which permits unrestricted use, distribution, and reproduction in any medium, provided the original author and source are credited.

Funding: The Rowett Institute receives funding from SG-RESAS (Scottish Government Rural and Environmental Science and Analysis Service). Visit of M.V. was supported by research grants from FEMS and Slovene human resources development and scholarship funds. Parts of this work were funded by grants from the United States-Israel Binational Science Foundation (BSF), Jerusalem, Israel - BSF Energy Research grant to E.A.B. and B.A.W. and Regular BSF Research grants to R.L. and B.A.W. - and by the Israel Science Foundation (grant nos 966/09 and 159/07 291/08). The funders had no role in study design, data collection and analysis, decision to publish, or preparation of the manuscript.

Competing Interests: The authors have declared that no competing interests exist.

*E-mail: h.flint@abdn.ac.uk.

\section{Introduction}

Bacteria related to Ruminococcus flavefaciens play an important role in the degradation of lignocellulosic plant fibre in the rumen and large intestine of herbivorous animals. Many R. flavefaciens strains isolated from the rumen are able to degrade insoluble crystalline cellulose present in test substrates such as filter paper and dewaxed cotton fibre. Studies over the past 10 years have demonstrated the presence of an elaborate cellulosome complex in this species [1,2]. Thus all cellulolytic strains so far examined possess the sca gene cluster that encodes cell surface scaffoldin proteins shown to bind the enzymatic subunits of the cellulosome via specific cohesindockerin interactions [3,4]. Information on the $R$. flavefaciens cellulosome has been greatly advanced by the genome sequence of strain FD1, which has revealed more than 200 putative cellulosome components $[5,6]$.

Many questions remain unanswered, however, not only concerning cellulosome organization but more generally about functional aspects of lignocellulose degradation by $R$. flavefaciens. One of the key differences between the cellulosome of $R$. flavefaciens 
and that of other cellulolytic species is the lack of an identified cellulose-binding module within the major cellulosomal scaffolding proteins of $R$. flavefaciens [4]. Instead, binding to cellulose has been assumed to be mediated via modules present in individual enzymes [7] and by the surface-attached protein CttA [8], but critical evidence on substrate binding by whole cells, as opposed to recombinant protein modules, has been lacking. Earlier work reported a mutational variant of $R$. flavefaciens strain $007 \mathrm{C}$ (referred to as $007 \mathrm{~S}$ ) that had lost the ability to degrade dewaxed cotton cellulose, while largely retaining the ability to degrade other forms of insoluble cellulose such as Avicel [9,10]. This difference was thought likely to reflect differences in substrate adhesion resulting from changes in extracellular protein expression that potentially identify the key proteins involved [8]. Cellulosomal components are known to be subject to regulation in $R$. flavefaciens, since growth on cellulose compared to cellobiose leads to increased transcription of several cellulosomal scaffolding proteins and enzymatic subunits in strain FD1 [5]. Information on the abundance of individual proteins and on substrate-driven changes in extracellular protein expression has however been somewhat limited $[8,11]$.

This investigation examines the extracellular proteomes of $R$. flavefaciens strains $007 \mathrm{C}$ and $007 \mathrm{~S}$ grown with crystalline cellulose (Avicel), xylan or cellobiose as energy sources. Our results reveal the up-regulation of several major cellulosome-associated proteins during growth on cellulose, consistent with evidence from the previous transcriptional studies in $R$. flavefaciens strain FD1 [5]. In addition however we detect major increases in a pilus-associated protein and in rubreryrthin in response to cellulose, and multiple differences in protein expression between the strain $007 \mathrm{C}$ and $007 \mathrm{~S}$ that suggest new requirements for effective cellulose degradation in this species, including the likely involvement of type IV pili.

\section{Results}

\section{Major Extracellular Proteins of $R$. flavefaciens $007 C$ and $007 \mathrm{~S}$}

Ruminococcus flavefaciens 007 was first isolated from rumen fluid as an actively cellulolytic culture able to degrade dewaxed cotton cellulose. This activity was lost upon subculture with cellobiose as the energy source, resulting in cotton-degrading $(007 \mathrm{C})$ and nondegrading (007S) derivatives $[9,10]$. In the present study we investigated the major extracellular proteins produced by each of these strains during growth on alternative substrates. Ruminococcus flavefaciens $007 \mathrm{C}$ cultures were grown anaerobically on modified Hungate-Stack medium containing $0.4 \%$ cellobiose, $0.4 \%$ oat spelt xylan, $1 \%$ insoluble cellulose (Avicel PH101) or $0.1 \%$ dewaxed cotton as added energy sources. Strain 007S cannot grow on de-waxed cotton cellulose, but its growth with cellobiose, Avicel and oat spelt xylan is similar to that of 007C (Fig. S1, [9]). Cultures were harvested at two time points in stationary growth phase and processed as described in the Materials and Methods section to give concentrated cell culture supernatant (CCSUP), bacterial cell wall-associated (CWAP) and cellulose-bound (CBP) cell fractions. Fractions from two independent experiments were analyzed for each strain.

Major spots of the three fractions were excised after 2D gel separation of proteins from cellulose-grown cultures (Fig. 1) and identified by mass spectrometry (ESI-MS/MS); identities are listed in Tables S1-S5. Four of the five proteins encoded by the sca gene cluster (ScaA, ScaB, ScaC and CttA) were among the most prominent proteins detected in the culture supernatant from Avicel-grown cultures. A draft genome sequence obtained for $R$. flavefaciens $007 \mathrm{C}$ showed that the sca genes are arranged in the same order as in strains FD-1 and 17 [3]. The predicted sca gene products show close to $100 \%$ sequence identity with those of $R$. flavefaciens 17 (Table 1).

In addition enzymes carrying GH48, GH9 and GH26 catalytic domains as well as type 1 dockerin domains (Doc-1) were identified among the major proteins in culture supernatants. These proteins show a close resemblance to homologues from $R$. flavefaciens strain FD-1. The catalytic domains also showed a high degree of amino acid sequence identity $(>40 \%)$ with major extracellular enzymes from $R$. albus that lack dockerins (Fig. 2). Additional proteins identified in the three fractions are listed in Tables S1-S5, and several of these are discussed further below.

\section{Influence of Growth Substrate on Expression of Major Extracellular Proteins}

We detected strong up-regulation of three proteins in culture supernatants of strain $007 \mathrm{C}$ (>10 fold) that was specific to Avicelgrown relative to cellobiose-grown cultures, with little or no increase in xylan-grown cultures (Fig. 3, 4A). These proteins were a GH48 cellulase, a type IV pilin homologue and a rubrerythrinlike protein; in contrast, the cellulosomal proteins ScaC (Fig. 3) and GH9 and GH26 enzymes (Fig. 3, 4A) were less affected by the growth substrate. There was some evidence for substratedependent expression of CttA and ScaA, especially among cell wall associated proteins (Fig. S2), but these appeared to increase in both xylan and Avicel-grown cultures relative to cellobiose.

\section{Comparison of Extracellular Protein Expression between 007C and 007S}

Protein expression was compared for the two strains $007 \mathrm{C}$ and 007S after the same period of growth on each substrate, based on the mean of two independent experiments. The strains did not differ significantly for the cell wall-associated protein fraction (Fig. S2). Differences were however detected for the culture supernatant fraction (Fig. 3, Fig. 4), including two of the proteins that showed the largest degrees of induction by Avicel in strain $007 \mathrm{C}$, the type IV pilin and rubrerythrin homologues. Interestingly the type IV pilin protein was also detected in the cotton-bound fraction of $R$. flavefaciens 007C (Fig. 1C, Table S4).

\section{Type IV pilin Gene Cluster in R. flavefaciens 007}

The 14-kDal protein identified in $R$. flavefaciens supernatant fluids shows N-terminal homologies with type IV pilins of several Gram-negative bacteria (Moraxella bovis, Pseudomonas aeruginosa, Dichelobacter nodosus, Neisseria meningitidis, Myxococcus xanthus, Eikenella corrodens, Neisseria gonorrhoeae in Aeromonas hydrophila, Deinococcus radiodurans, Vibrio vulnificus, Photobacterium profundum) (Fig. S3). This protein also showed $55.6 \%$ amino acid sequence identity with pilA2 from Ruminococcus albus 20. From the location of prepilin peptidase recognition motif ( $\hat{G} F x x=$ ) detected in the sequence of $R$. flavefaciens 007 pilin, it is possible to infer that the protein is synthesised with a 7-residue leader sequence (MKTTKKG), that is cleaved to release a 165-residue mature polypeptide with a theoretical pI of 5.75 and MW of 13435 Da. These values do not correspond exactly with the position observed on the $2 \mathrm{D}$ gel of supernatant protein fraction, probably indicative of post-translational modification (PTM), as reported for the $R$. albus pilin $[12,13]$. The shift toward higher MW that is accompanied by lowering of protein $\mathrm{pI}$ is often associated with the addition of phosphate groups, which was supported by NetPhosK analysis of Pil3 sequence revealing 17 possible phosphorylation sites. Interestingly, the properties of the same protein in CBP fraction appear 

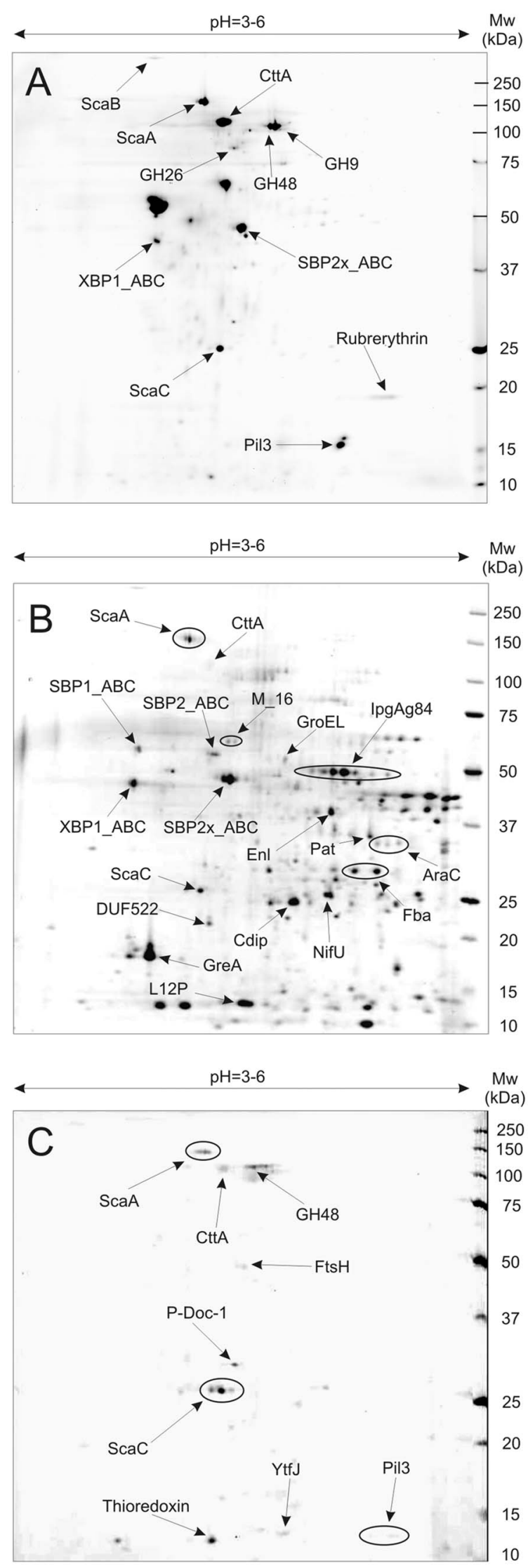

PLOS ONE | www.plosone.org
Figure 1. Proteome of cellulose-grown Ruminococcus flavefaciens $007 \mathrm{C}$. Showing 2D gel separations of: A) cell culture supernatant (CCSUP) protein fraction, B) cell wall associated (CWAP) fraction (following growth on Avicel for 7.5 days); C) cellulose-bound (CBP) fraction following growth on dewaxed cotton for 9.5 days. Abbreviations: Pil3 - protein with type IV pilin N-terminal domain (encoded by pil3), P-Doc-1 - protein with dockerin type-1 (no other conserved domains/signature sequences have been detected), GH - glycoside hydrolase, SBP_ABC - substrate-binding component of ABC-type sugar transport system, XBP_ABC - xylose-binding component of ABC-type sugar transport system, SBP2x_ABC - substrate-binding component of ABC-type sugar transport system involved in xylan utilization, M_16Zn-dependent peptidase, IpgAg84 - immunogenic protein antigen 84, GroEL - GroEL chaperonin, Enl - enolase, Pat - phosphate acetyltransferase, Fba - fructose-bisphosphate aldolase, NifU - NifU homolog involved in Fe-S cluster formation, GreA - transcription elongation factor GreA, Cdip - cell division initiation protein, DUF552 - putative conserved protein with unknown function (DUF552), AraC - transcriptional regulator, $\mathrm{L} 12 \mathrm{P}$ - 50S ribosomal protein $\mathrm{L} 12 \mathrm{P}, \mathrm{YtfJ}$ - sporulation protein YtfJ, FtsH - ATP-dependent metalloprotease. doi:10.1371/journal.pone.0065333.g001

to be somewhat closer to the calculated values, indicating lower level (or absence) of PTMs. This may affect protein polymerization and formation of visible structures, as in the case of $\mathcal{N}$. meningitidis. In these bacteria, the major subunit of type IV pili is posttranslationally modified by the addition of phosphoglycerol at Ser93, which results in increasing its negative charge leading to destabilization of the pili and detachment of bacteria from the microcolonies on host tissue in certain periods of growth cycle [14].

The $R$. flavefaciens pil3 gene is flanked by open reading frames that are homologous to genes known to be involved in the assembly and secretion of type IV pili and flagella (Fig. 5, Table S6). These include upstream genes encoding a putative pilus retraction (PilT-like) ATPase, followed by a PilB (Vir B11)-like ATPase, and a putative prepilin peptidase (PilD-like) gene. The first two genes in the cluster encode a putative PilM-like pilus assembly protein (involved in the export of the pilus subunit and its assembly in $P$. aeruginosa) and a putative F-domain of the type II secretion system (PulF homologue). One gene (pil4) with a type IV pilus signature sequence (prepilin-type N-terminal cleavage/ methylation site) is present downstream of pil3, and also encodes a protein with high similarity to $R$. albus pilin CbpC. The nucleotide composition of the putative pilus biogenesis cluster did not differ significantly in its $\mathrm{G}+\mathrm{C}$ content $(47.04 \%)$ from the whole genome $(45.2 \%)$. We also detected a homologous gene cluster in the genome of $R$. flavefaciens FD1 [5].

Figure 6 shows the morphology of cells of $007 \mathrm{C}$ and $007 \mathrm{~S}$ grown on cellobiose or cellulose as revealed by scanning EM. Contact between the bacterial extracellular matrix and the cellulose surface is evident particularly for strain $007 \mathrm{C}$.

\section{Discussion}

The sca gene cluster of $R$. flavefaciens $007 \mathrm{C}$ is almost identical to that reported in $R$. flavefaciens 17 [4], suggesting that this strain will show a similar cellulosome organization. The proteomic analysis conducted here reveals that four sca-encoded proteins, ScaA, ScaB, CttA and ScaC, are among the major supernatant and cellassociated proteins detected in Avicel-grown cultures. The fifth sca protein $(\mathrm{ScaE})$ is covalently bound to the bacterial cell wall in the closely related $R$. flavefaciens strain 17 and would therefore not be expected to appear in the fractions examined here [11]. In addition, three dockerin-carrying enzymes, a GH48, GH9 and GH26, were prominent in culture supernatant fluids of Avicelgrown cells. Up-regulation of the major exo-acting GH48 has also 


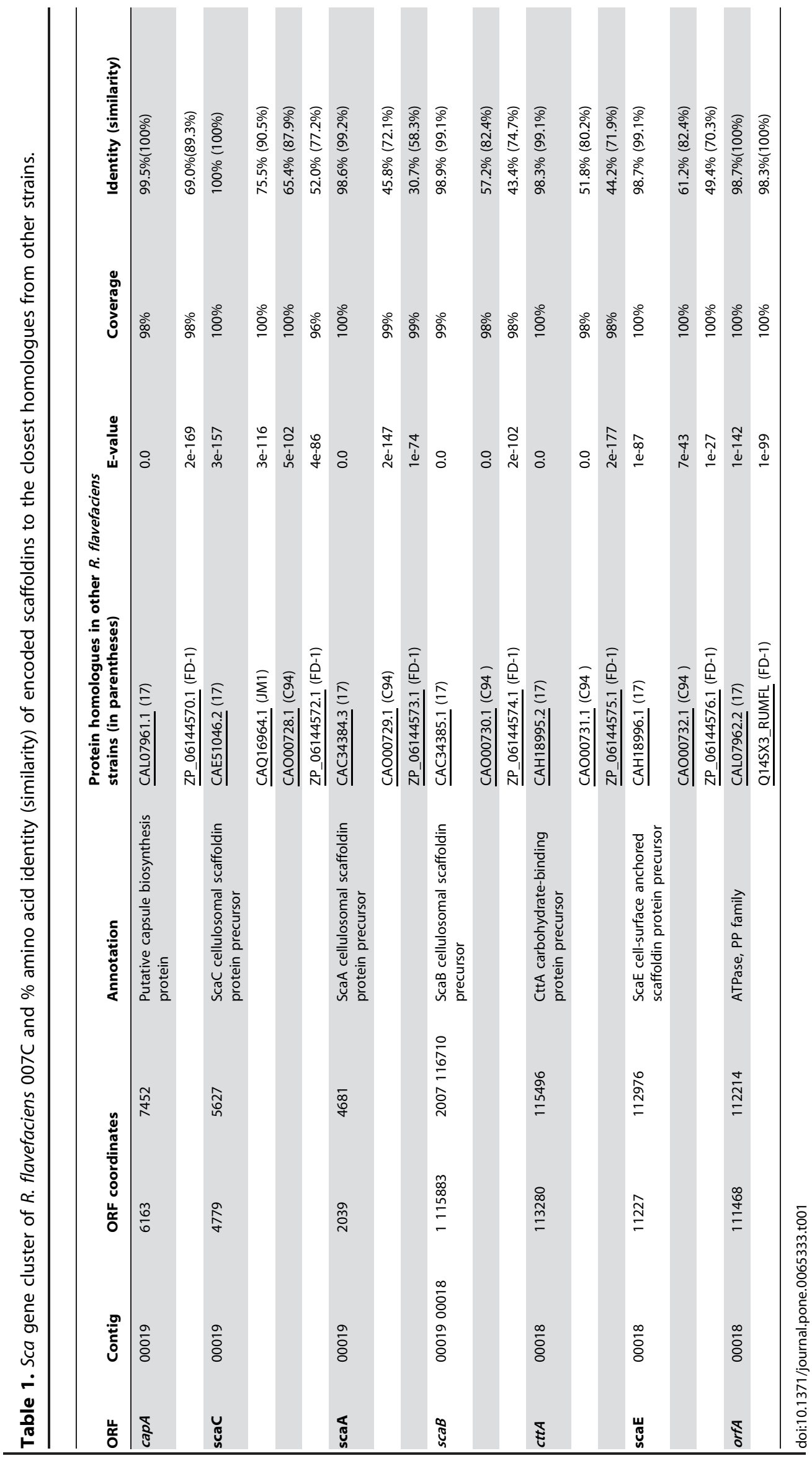



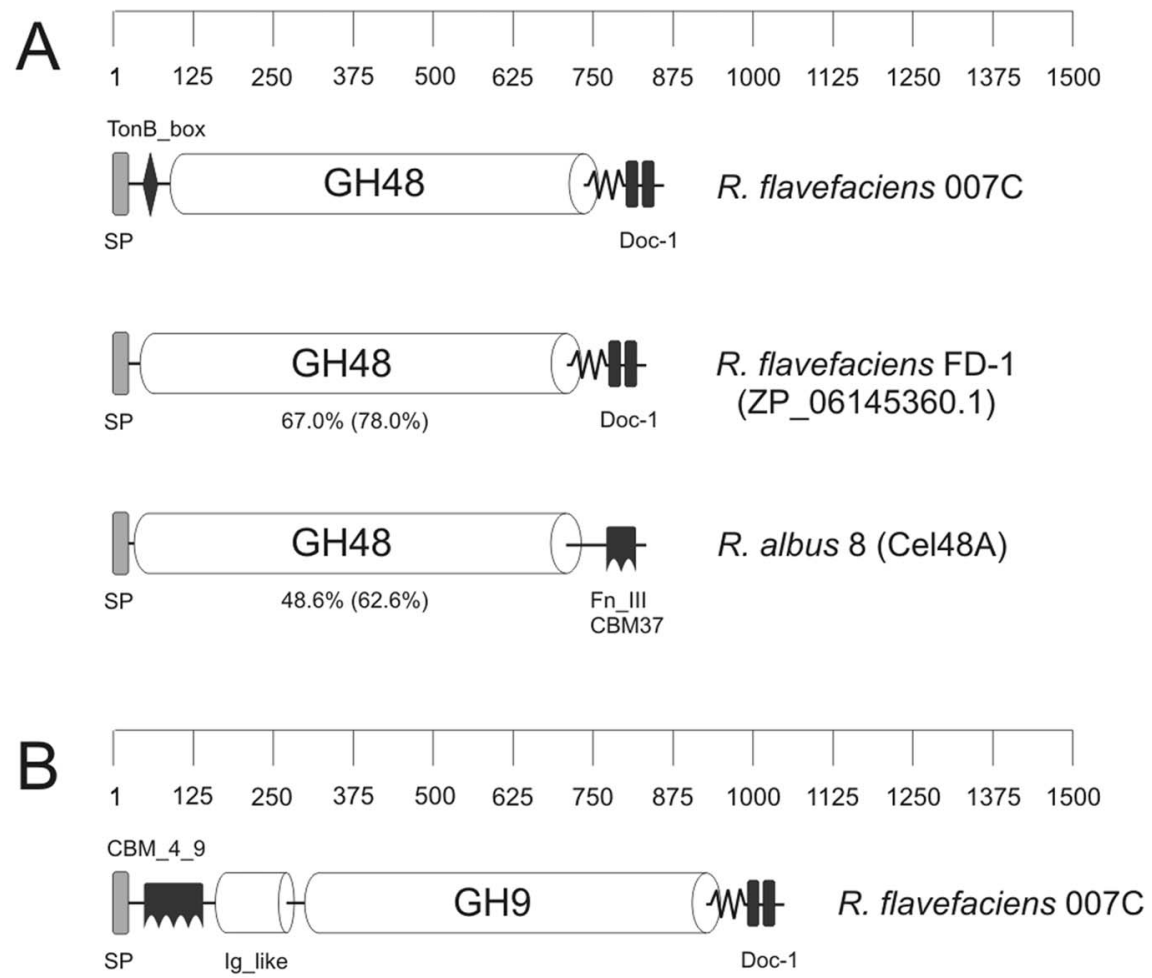

CBM_4_9
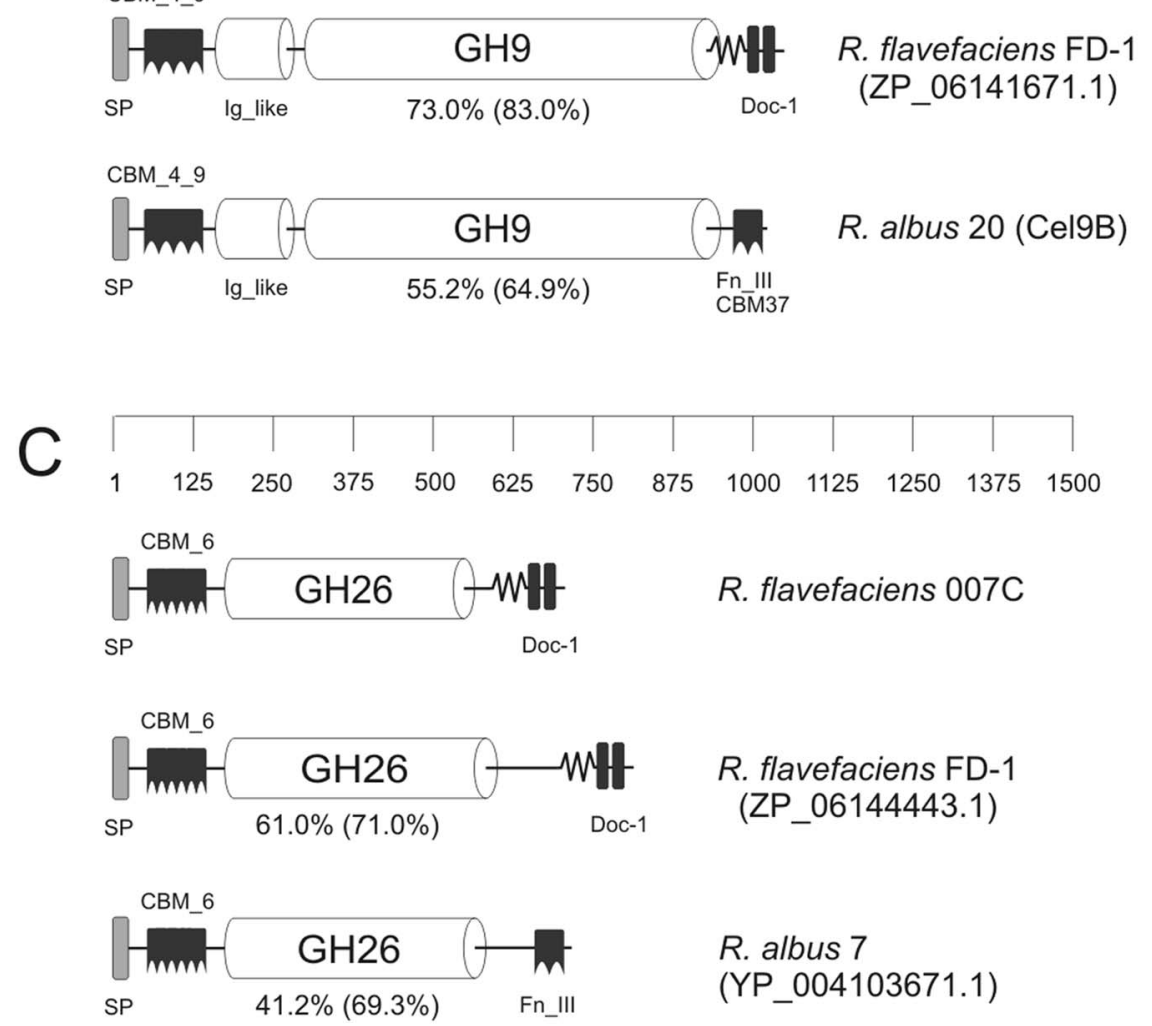

Figure 2. Modular structures of prominent glycoside hydrolases. These are compared with the predicted enzymes showing the highest $\%$ similarity (Blast) from R. flavefaciens FD-1 or R. albus: (A) glycoside hydrolase family 48; (B) glycoside hydrolase family 9; (C) glycoside hydrolase family 26. Abbreviations: SP - signal peptide, Doc-1 - dockerin type I, GH - glycoside hydrolase, CBM - carbohydrate-binding module, TonB_box: TonBdependent receptor protein signature 1, Fn_III - fibronectin type III domain, Ig-like - immunoglobulin-like domain. The numbers below the $R$. flavefaciens FD-1 and $R$. albus enzymes represent \% amino acid identity to the $R$. flavefaciens $007 C$ homologue. doi:10.1371/journal.pone.0065333.g002 


\section{C}

AVICEL
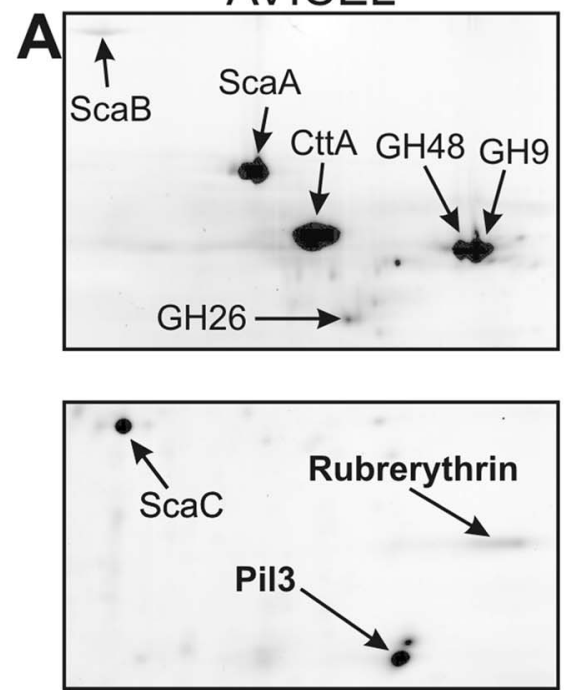

AVICEL
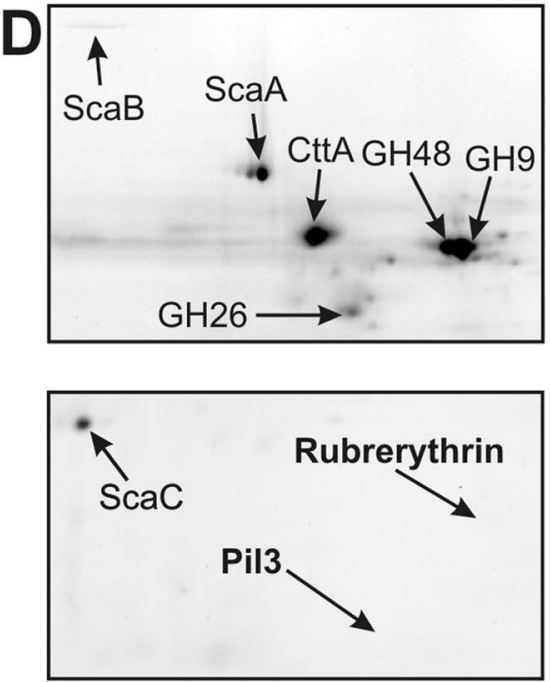

CELLOBIOSE
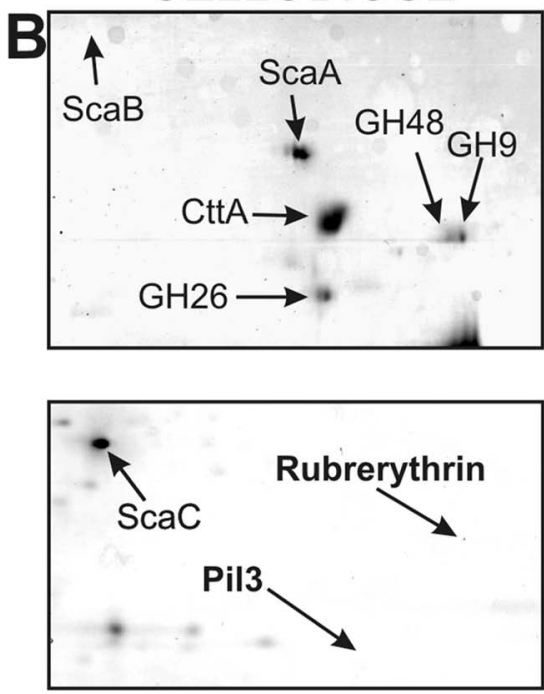

007S

CELLOBIOSE
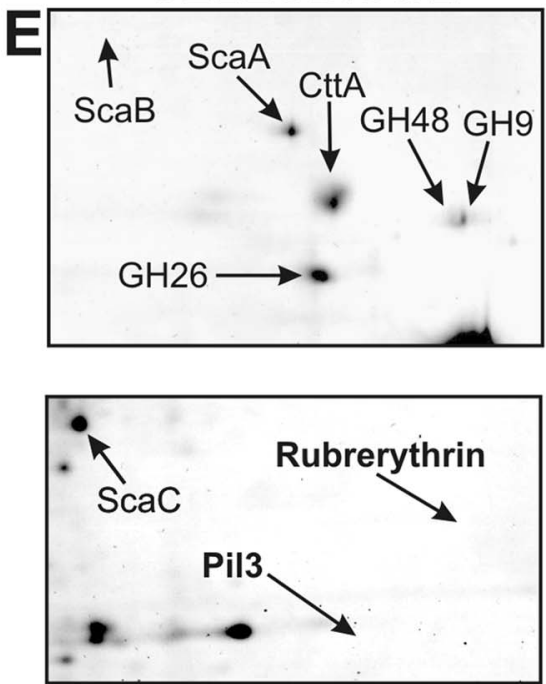

XYLAN
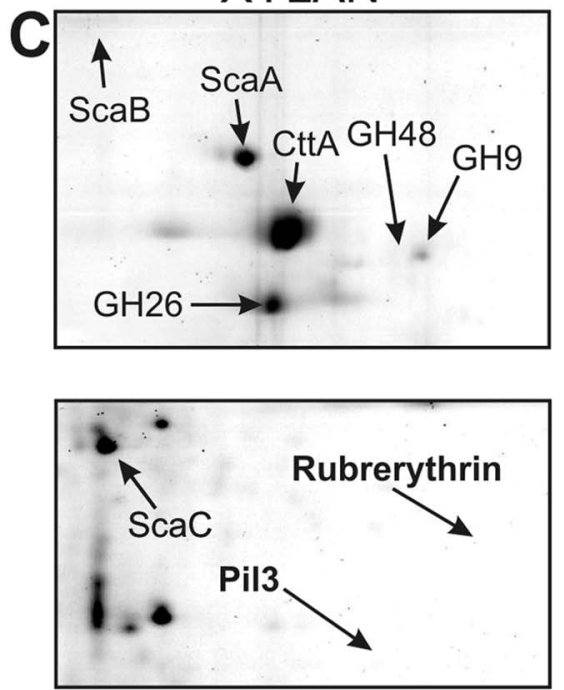

XYLAN
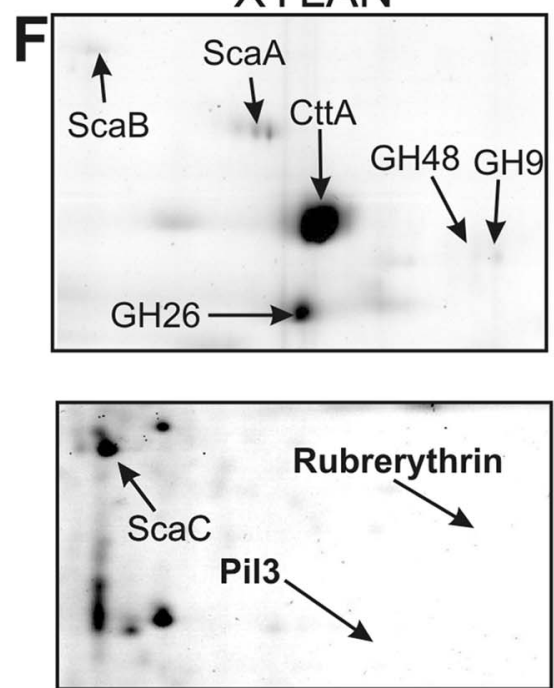

Figure 3. Impact of growth substrate on the extracellular proteome. Expanded views are show for 2D gel separations of supernatant proteins from R. flavefaciens $007 \mathrm{C}$ and $007 \mathrm{~S}$ cutures grown on Avicel (7.5 days), xylan (48 hours) or cellobiose (24 hours). The arrows point to identified proteins; relative expression levels for some of these are shown in Figure 4.

doi:10.1371/journal.pone.0065333.g003

been observed in C. thermocellum during growth on cellulose [1518] and this enzyme is known to play a key role in cellulose breakdown. A prominent GH44 enzyme present in the proteome of xylan-grown $R$. flavefaciens 17 [19], although encoded by the genome, was not detected here in $R$. flavefaciens 007 .

Regulation of sca cluster gene expression at the mRNA level has been examined in $R$. flavefaciens FD-1 by Berg Miller et al. [5] who reported approximately 4.5 fold up-regulation of $s c a A$, scaB and scaC mRNA in cells grown on cellulose in comparison to cellobiose. Our study suggests up-regulation of ScaA and ScaB proteins in $R$. flavefaciens $007 \mathrm{C}$, although $\mathrm{ScaC}$ was unaffected by the growth substrate. The previous study [5] also detected considerable up-regulation of several multimodular xylanases, but did not report upregulation of the GH48 enzyme that was seen here. The differences between the two studies might perhaps reflect inter-strain differences. On the other hand the previous study examined mRNA expression in cells at early growth stages with a different type of cellulose substrate $(9 \mathrm{~h}$ of growth on cellobiose and $19 \mathrm{~h}$ growth on filter paper) while we have reported protein levels in different cell fractions in stationary cultures (after $14 \mathrm{~h}$ growth on cellobiose and $180 \mathrm{~h}$ growth on Avicel). Differences in temporal gene expression, protein secretion and 

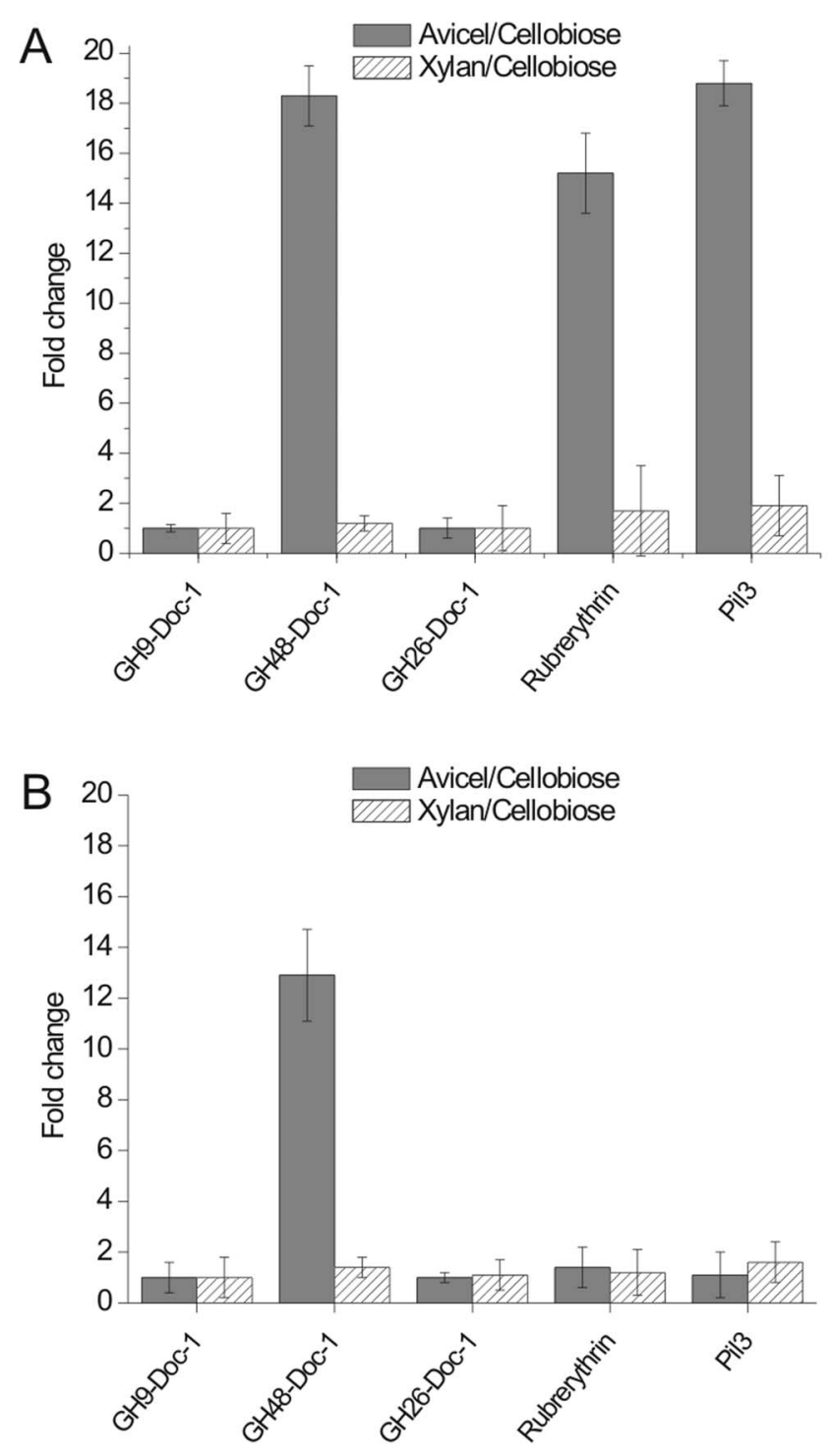

Figure 4. Cellulose-specific changes in expression of culture supernatant proteins in $R$. flavefaciens strains grown on Avicel (7.5 days), xylan (48 hours) or cellobiose ( 24 hours). (A) supernatant (CCSUP) fraction of strain 007C, (B) supernatant fraction of strain 007S. 1-fold change indicates no change, $>1$-fold change indicates upregulation in comparison to cellobiose-grown cultures, $<1$ fold change indicates downregulation in comparison to cellobiosegrown cultures. Results are based on duplicated biological experiments, and three technical replicates for each gel separation. doi:10.1371/journal.pone.0065333.g004

protein turnover could therefore all be involved in explaining these differences. Differential regulation in response to the growth substrate has also been reported recently for C. cellulovorans cellulosomal proteins [20].

An important new finding from this study is the detection of an abundant type IV pilin protein both in Avicel-grown culture supernatants and among proteins attached to cotton cellulose in $R$. flavefaciens $007 \mathrm{C}$. Furthermore this protein was specifically upregulated (19 fold) by growth of $R$. flavefaciens $007 \mathrm{C}$ on Avicel compared with cellobiose, suggesting that it has a role in cellulose breakdown. Type IV pili have also been shown to be expressed in the related cellulolytic species $R$. albus where they are reported to have a role in binding bacterial cells to cellulose [13,21-23].
Interestingly the evidence for this was based on studies on variants of two different strains of $R$. albus that had been selected for loss of adhesion to cellulose $[13,23]$. Similarly, a cellulose binding protein identified as type IV pilin was found to be missing in cellulose adhesion-defective mutants of Fibrobacter succinogenes [24]. Pili composed of type IVa subunits are found in a broad range of Gram-negative bacteria with disparate host and tissue specificity, but have also been reported from some Gram-positive species [25]. They apparently play different physiological roles: twitching motility, host cell adhesion and immune escape, microcolony formation and DNA uptake.

Another novel finding was the strong up-regulation of a protein annotated as a putative rubrerythrin-like protein during growth on Avicel. This $20-\mathrm{kDa}$ protein has a conserved rubredoxin-type $\mathrm{Fe}(\mathrm{Cys})_{4}$ site at the N-terminal region and a ferritin-like di-iron domain at the C-terminus. Rubrerythrin is a non-heme iron enzyme, widely distributed in anaerobic bacteria and archaea [2628]. Although it would normally be assumed to be intracellular, it accumulated in the supernatant fluids of $R$. flavefaciens $007 \mathrm{C}$ cultures and was strongly upregulated by growth on cellulose. Various activities of rubrerythrin-like proteins have been described in bacteria [29], including pyrophosphatase, ferroxidase and superoxide dismutase activities [26]. However, recent reports suggest that its true function may be as a $\mathrm{H}_{2} \mathrm{O}_{2}$ reductase as part of an alternative detoxification system in strict anaerobic bacteria and archaea [30]. Recently, expression of a small rubrerythrin-like protein in Clostridium acetobutylicum, Hsp21, was shown to be induced not only by oxidative stress, but also by various other stress factors, such as salt, increased $\mathrm{pH}$, high concentration of solvents or cold shock [31]. The possible significance of this rubrerythrin-like protein for growth of $R$. flavefaciens on cellulose is an intriguing question for future research.

The strain $007 \mathrm{~S}$ differs from $007 \mathrm{C}$ in its cell morphology as well as in its inability to grow on cotton cellulose, while retaining the ability to degrade Avicel [9,10]. Strain 007S has been shown here to display several quantitative protein expression changes by comparison with $007 \mathrm{C}$. In particular, two proteins, the type IV pilus and the rubrerythrin-like protein showed markedly lower upregulation in Avicel-grown relative to cellobiose-grown cultures of 007S compared with 007C. It seems probable that the loss of cotton cellulose degradation observed in $007 \mathrm{~S}$ results from a regulatory mutation that exerts pleiotropic effects that include reduced expression of key adhesion factors. A previous report indicated reduced expression of the substrate-binding protein CttA found in cellulose-attached proteins from 007S compared with that of strain 007C [11]. The present study however revealed little difference between $007 \mathrm{~S}$ and $007 \mathrm{C}$ in $\mathrm{CttA}$ expression among total supernatant proteins.

The fact that the cotton non-degrading variant $007 \mathrm{~S}$ showed reduced expression of the pil3 gene suggests that type IV pili, although yet to be demonstrated directly in this species, might play a special role in the degradation of highly crystalline cellulose by $R$. flavefaciens. Adhesion of $R$. flavefaciens to insoluble cellulosic substrates appears to involve multiple adhesion factors including type IV pili, specialist adhesion proteins (CttA) [7] and CBMs present in individual enzymes [8]. Multiple adhesion mechanisms have also been proposed in $R$. albus [32] although there has been no report of a CttA-like protein in that species. The relative importance of these different binding mechanisms is likely to vary with the nature of the substrate and the proximity of cells and enzyme complexes to the surface of the substrate. Since the $007 \mathrm{~S}$ variant is still able to degrade Avicel, this suggests that type IV pili could be particularly important in adhesion to highly crystalline celluloses such as cotton cellulose. 


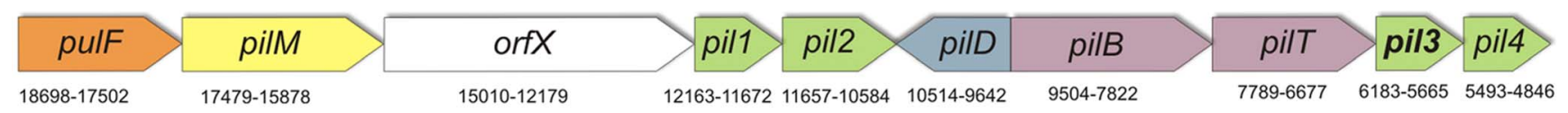

Figure 5. Type IV pili biogenesis cluster of $\boldsymbol{R}$. flavefaciens $\mathbf{0 0 7 C}$. The protein Pil3, encoded by the pil3 gene, shares $51 \%$ amino acid identity to the cellulose-binding protein (CbpC) of $R$. albus 8 and $43 \%$ amino acid identity to $R$. albus 20 protein GP25 (a protein that was shown to be underproduced by spontaneous cellulose adhesion-defective mutant D5). The gene downstream (pil4) encodes another protein with prepilin-type IV $\mathrm{N}$-terminal domain, whereas the genes upstream encode: putative PilT-/PilB-like ATPases (pilT, pilB), prepilin peptidase (pilD), two proteins with pseudopilin PulG-like domains (pil1, pil2), type IV pilus assembly protein (pilM) and a type II secretion system protein (pilf). OrfX refers to open reading frame without recognized signal sequences.

doi:10.1371/journal.pone.0065333.g005

In conclusion this study has used proteomic analysis to examine the expression of major extracellular proteins relevant to lignocellulose breakdown in $R$. flavefaciens 007. Our findings identify the major cellulosome-associated structural and catalytic proteins within the extracellular proteome, but also reveal for the first time a likely role for type IV pili in cellulolysis by $R$. flavefaciens. These appendages are proposed to play a role in the adhesion to cellulose by $R$. albus, wherein there has been no direct evidence for cellulosome organization, and the type IV pili are now candidates for contributing to cellulose binding also in the cellulosomal species, $R$. flavefaciens.

\section{Materials and Methods}

\section{Strains and Growth Conditions}

$R$. flavefaciens $007 \mathrm{C}$ and $007 \mathrm{~S}$ were grown anaerobically in $800 \mathrm{ml}$ of Hungate-Stack medium [33] modified by the addition of $5 \%$ clarified rumen fluid. Medium was supplemented with $1 \%$ (wt/vol) Avicel PH101 (Honeywill \& Stein, London, UK), 0.4\% oat spelt xylan, $0.4 \%$ cellobiose (Sigma-Aldrich) or $0.1 \%$ (wt/vol) dewaxed cotton as energy sources. The cells were grown statically at $37^{\circ} \mathrm{C}$ until the beginning of stationary phase. Extracellular proteins were harvested at two or three time points of incubation
A

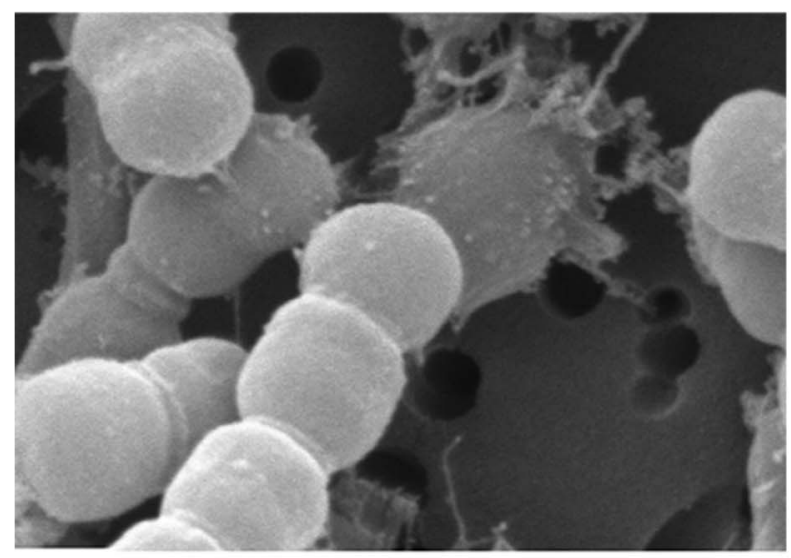

C

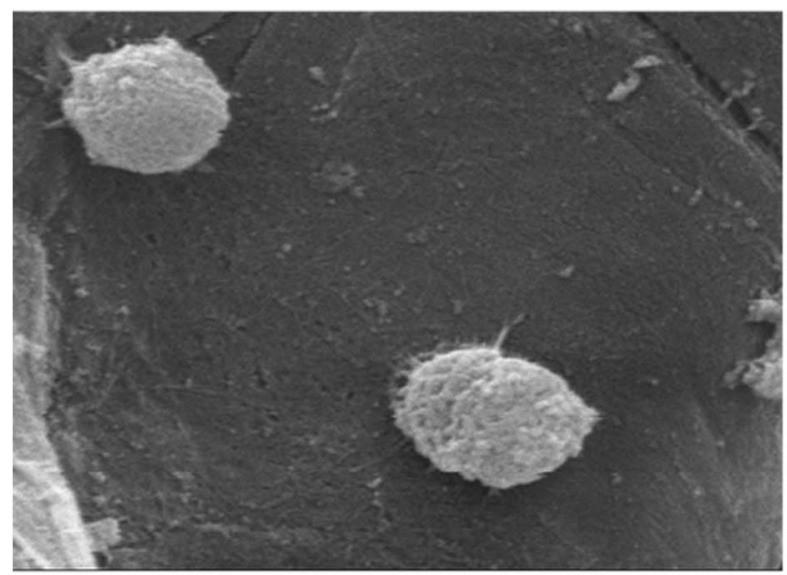

$300 \mathrm{~nm}$
B

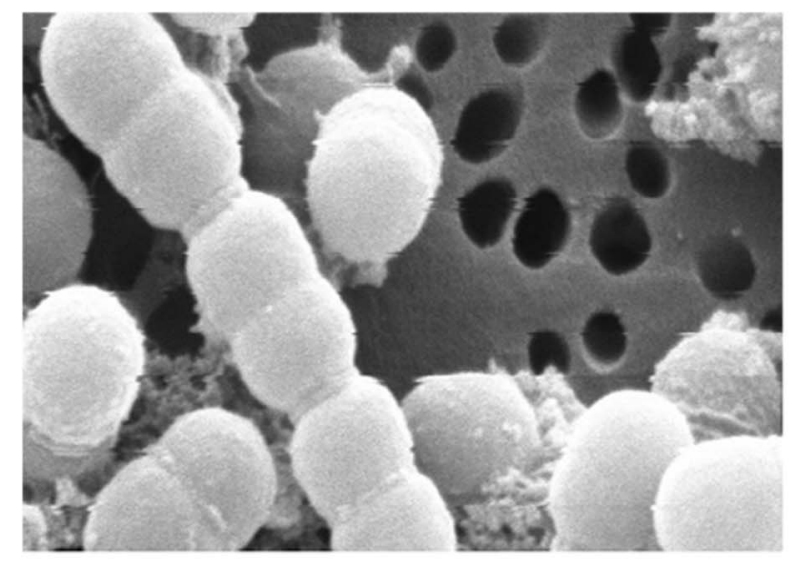

D

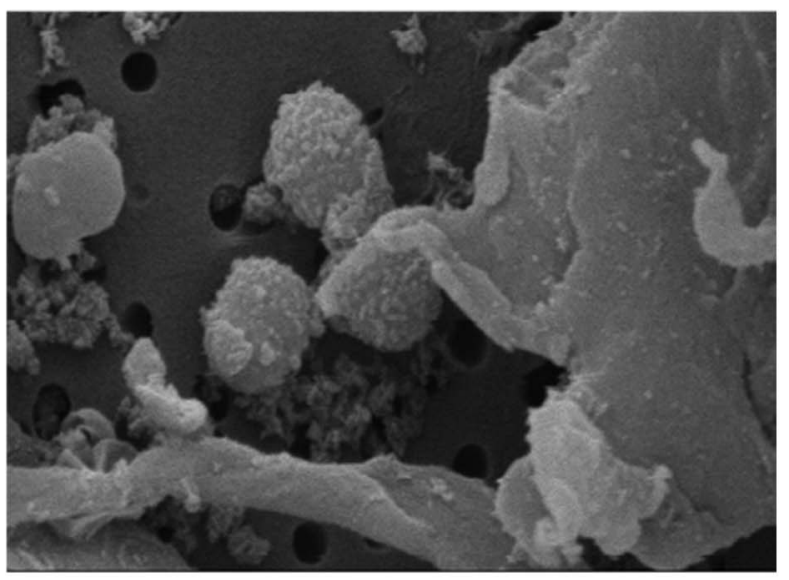

Figure 6. $\boldsymbol{R}$. flavefaciens 007 cell morphology revealed by scanning electron microscopy. Cells grown on cellobiose: (A) 007C, (B) $007 \mathrm{~S}$; or on cellulose: (C) 007C, D) 007S.

doi:10.1371/journal.pone.0065333.g006 
on each substrate: after 14 and 24 hours of growth on cellobiose, 26 and 48 hours of growth on oatspelt xylan, 7.5 and 9.5 days of growth on Avicel and after 9.5, 11.5 and 13.5 days of growth on dewaxed cotton, which corresponded to the periods when highest specific avicelase activity and total protein concentration was measured during growth of the cultures (procedure below, Fig. S1).

\section{Total Protein and Avicelase Activity}

To estimate the growth and cellulase activity on different substrates, the $R$. flavefaciens strains $007 \mathrm{C}$ and $007 \mathrm{~S}$ were grown in duplicates of $8 \mathrm{ml}$ modified Hungate-Stack supplemented with carbon sources as described above. After chosen periods of growth (between 10-48 hours for cultures grown on cellobiose, 10-78 hours for cultures grown on xylan and 1-15 days for cultures grown on cellulose substrates) the tubes were centrifuged at $9000 \mathrm{~g}$, at $4^{\circ} \mathrm{C}$, and the supernatants were separated from the pellets and frozen following the addition of $80 \mu \mathrm{L}$ of protease inhibitors cocktail (PIC; Sigma Chem. Co., St. Louis MO). The pellets were washed with $2 \mathrm{ml}$ of $50 \mathrm{mM}$ Na-phosphate buffer pH $6.5(\mathrm{NaPB})$, added $50 \mu \mathrm{l}$ of PIC and resuspended in $1 \mathrm{ml}$ of sterile MilliQ. To determine the rate of Avicel degradation by different fractions, $40 \mu \mathrm{l}$ aliquot of each supernatant or pellet each sample was mixed with $230 \mu \mathrm{l}$ of $1 \%$ (w/v) Avicel suspension in NaPB containing $1 \mathrm{mM} \mathrm{CaCl}_{2}$ and incubated $120 \mathrm{~min}$ at $37^{\circ} \mathrm{C}$. After incubation, the reaction was stopped by $30 \mu \mathrm{l}$ of $10 \%$ TCA and the mixture was centrifuged at 10,000 g. The concentration of reducing sugars in the supernatant was measured by the Lever method [34] using glucose as a standard. Total protein concentrations were determined by the method of Bradford using the Coomasie Plus kit (Pierce, Thermo Scientific).

\section{Extracellular Protein Fractionation}

Cell culture supernatant fluids were removed after centrifuging the cultures at $10,000 \mathrm{~g}\left(4^{\circ} \mathrm{C}\right.$ for $\left.15 \mathrm{~min}\right)$, then dialyzed (four times against $4 \mathrm{~L}$ of distilled water, at $4^{\circ} \mathrm{C}$ ), freeze dried and resuspended in $10 \mathrm{ml}$ of distilled water $\left(\mathrm{dH}_{2} \mathrm{O}\right)$. Cell culture supernatant protein fraction (CCSUP) was then stored at $-20^{\circ} \mathrm{C}$ pending analysis. Cell wall-associated protein (CWAP) and cellulose-bound protein $(\mathrm{CBP})$ enriched fractions were prepared by slight modifications of previously published procedures [11,35]. When preparing the cultures grown on cellulose, the cells were first separated from the substrate by differential settling. Following vigorous shaking, the substrate was left to sediment for $10 \mathrm{~min}$. The cell culture supernatant fluid (containing detached cells) was then removed using a vacuum pump and centrifuged at 10,000 g, $4^{\circ} \mathrm{C}$ for $15 \mathrm{~min}$. Cell pellets were washed twice with $100 \mathrm{ml}$ TBS (25 mM Tris-HCl, $150 \mathrm{mM} \mathrm{NaCl}, \mathrm{pH} 7.0)$ and centrifuged at $10,000 \mathrm{~g}\left(4^{\circ} \mathrm{C}, 15 \mathrm{~min}\right)$. The pellet from the final centrifugation step was resuspended in $40 \mathrm{ml}$ of $4.5 \mathrm{mM} \mathrm{MgSO}_{4}$ in TBS with the addition of $2 \mu \mathrm{g}$ DNAse I and RNAse $\mathrm{A} \mathrm{ml}^{-1}$ and incubated at room temperature for $45 \mathrm{~min}$. The pellet from another centrifugation $\left(10,000 \mathrm{~g}, 4^{\circ} \mathrm{C}, 15 \mathrm{~min}\right)$ was resuspended in $14 \mathrm{ml}$ of sarcosyl buffer ( $2 \% \mathrm{~N}$-lauryl sarcosine, $1 \mathrm{mM}$ protease inhibitor cocktail (Sigma Chem. Co., St. Louis MO), 1 mM PMSF (Sigma) in TBS) and incubated on ice for $1 \mathrm{~h}$ (with 3 short vortexing steps). After centrifugation at $15,000 \mathrm{~g}\left(4^{\circ} \mathrm{C}, 15 \mathrm{~min}\right)$ the supernatant was recovered and ultracentrifuged at 75,000 g, $4^{\circ} \mathrm{C}$ for $1 \mathrm{~h}$ (Optima Max ultracentrifuge, MSL-50 rotor, Beckman-Coulter, Fullerton, CA). This final supernatant fraction was $100 \times$ concentrated by ultrafiltration (Amicon-15, Millipore Inc., Billerica). The retentate (CWAP) was stored at $-20^{\circ} \mathrm{C}$ pending analysis.

To obtain the CBP fraction residual Avicel/cotton was vigorously washed five times with $40 \mathrm{ml}$ of TBS-Ca-Tween buffer (25 mM Tris-HCl, $150 \mathrm{mM} \mathrm{NaCl}, 1 \mathrm{mM} \mathrm{CaCl}$; pH 7.0). CBPs were recovered by incubating washed cotton/Avicel with $2 \%$ (w/ vol) CHAPS at $70^{\circ} \mathrm{C}$ for $1 \mathrm{~h}$, followed by heating at $100^{\circ} \mathrm{C}$ for 5 min. Supernatant fluids were $100 \times$ concentrated by ultrafiltration (Amicon-15) and stored at $-20^{\circ} \mathrm{C}$ pending analysis.

Two Dimensional Gel Electrophoresis and Image Analysis

All results relating to protein expression presented in this paper are based on duplicated biological experiments, with three technical replicates for each gel separation. Protein concentrations of CCSUP and CWAP fractions were measured by the BCA assay (Pierce Chem. Co., Rockford, IL). An aliquot of each sample containing $350 \mu \mathrm{g}$ of protein was mixed with isoelectric focusing buffer (9 M urea, 4\% CHAPS, 0.5\% Biolite Ampholite; pH 3-10) and used for rehydration of $17 \mathrm{~cm}$ IPG strips, $\mathrm{pH}$ 3-6. Rehydration was performed on a Bio-Rad IEF cell at $20^{\circ} \mathrm{C}$ for $1 \mathrm{~h}$ without applied voltage, then at $50 \mathrm{~V} / \mathrm{strip}$ for another $16 \mathrm{~h}$. Initial start-up and ramping was performed according to the BioRad instruction protocol. IPG strips were incubated in equilibration buffer I $(6 \mathrm{M}$ urea, 2\% SDS, $0.375 \mathrm{M}$ Tris- $\mathrm{HCl}$, $\mathrm{pH} 8.8,20 \%$ glycerol, $130 \mathrm{mM}$ dithiothreitol) and then in equilibration buffer II (6 M urea, 2\% SDS, 20\% glycerol, $135 \mathrm{mM}$ iodoacetamide, $0.375 \mathrm{M}$ Tris-HCl; $\mathrm{pH} 8.8$ ) for $15 \mathrm{~min}$ at room temperature. Gradient (8-16\%) SDS PAGE gels were prepared and cast on an Anderson IsoDalt casting apparatus. The gels were run at $200 \mathrm{~V}$ for $9.5 \mathrm{~h}$, then fixed and stained by Colloidal Coomasie Blue staining as previously described [19]. The gels were imaged with a Bio-Rad GS-800 Scanning Densitometer and then analysed with Progenesis Samespots software (Nonlinear Dynamics) with incorporated statistics package). Differences in protein expression were considered significant when the following criteria were met: 1) differences in average normalized spot volumes were $>5$-fold and Anova $\mathrm{p}$-values $<0.05$; or 2) differences in average normalized spot volumes were $>1.5$ and $\mathrm{p}$-values $<0.025$.

\section{Spot Cutting, Trypsinization, Protein Identification by NANO LC MS/MS}

The spots of interest (average spot volumes $>5 \mathrm{e}^{+006}$ ) were excised from the gels manually, placed in a 96-well V-bottom plate and trypsinized by using MassPrep station. The samples were analyzed using a nano LG system (LC Packings, Camberly, Surrey, UK) consisting of an 'Ultimate' nano LC system, with a column flow rate of $0.3 \mu \mathrm{L} \mathrm{min}{ }^{-1}$, a 'Famos' autosampler set to an injection volume of $10 \mu \mathrm{L}$ and a 'Switchos' microcolumn switching device with a flow rate $0.03 \mathrm{~mL} / \mathrm{min}$. The solvent used by the 'Switchos' was $0.1 \%$ formic acid. The nanocolumn was a C18 PepMap 100, $15 \mathrm{~cm} \times 75 \mu \mathrm{m}$ i.d., $3 \mu \mathrm{m}, 100 \AA$ (LC Packings). HPLC grade solvents were used, $2 \%$ acetonitrile and $0.1 \%$ formic acid (A) and $80 \%$ acetonitrile and $0.08 \%$ formic acid (B). The gradient started at $5 \% \mathrm{~B}$, proceeding to $95 \% \mathrm{~B}$ over $32 \mathrm{~min}$, maintained for $10 \mathrm{~min}$, then restored to $5 \% \mathrm{~B}$ for an additional $18 \mathrm{~min}$ giving a total run time of $60 \mathrm{~min}$. The system was equilibrated at $95 \% \mathrm{~A}$ for $3 \mathrm{~min}$ prior to injection of subsequent samples.

The mass spectrometry was performed using a Q-Trap (Applied Biosystems/MDS Sciex, Warrington, UK) triple quadrupole fitted with a nanospray ion source, where Q3 is operated as a linear ion trap (LIT). The nanospray needle voltage was set at $2800 \mathrm{~V}$. Oxygen Free Nitrogen (OFN) was used as the curtain gas and the collision gas. In the survey scan mode, the mass range in $\mathrm{Q} 1$ was set to $\mathrm{m} / \mathrm{z} 450-1450$ with a scan rate of $4000 \mathrm{amu} / \mathrm{s}$. The criteria for selection of ions for fragmentation (Q2) were ions of $1.5^{4} \mathrm{cps}$ or above. The collision energy was compound dependent (set to a 
maximum of $80 \mathrm{eV}$ ). The trap fill time (Q3) was set to a maximum of $250 \mathrm{~ms}$ and the scan rate was $4000 \mathrm{amu} / \mathrm{s}$.

\section{R. flavefaciens 007C Genome Sequence}

The $R$. flavefaciens $007 \mathrm{C}$ genome was sequenced with $55 \times$ coverage and assembled in 39 contigs by the Wellcome Trust Sanger Institute. The sequence is publicly available and was downloaded from their web site: ftp://ftp.sanger.ac.uk/pub/ pathogens/Ruminococcus/flavefaciens/007c/Rflav007c.091211. seq.

\section{Protein Identification and Data Analysis}

The total ion current (TIC) data were submitted for NCBInr database searching using the MASCOT search engine (Matrix Science) with the following search criteria: allowance of 0 or 1 missed cleavages; peptide mass tolerance of $\pm 1.5 \mathrm{Da}$; fragment mass tolerance of $\pm 1.5 \mathrm{Da}$, trypsin as digestion enzyme; carbamidomethyl fixed modification of cysteine; methionine oxidation as a variable modification; and charged state as $2^{+}$ and $3^{+}$. Identified proteins from different $R$. flavefaciens strains (mainly FD-1 and N17) were used to query the $R$. flavefaciens $007 \mathrm{C}$ local database for ORFs encoding orthologues in this strain (http://www.ncbi.nlm.nih.gov/projects/gorf/). Protein samples with MASCOT scores lower than 100 , were subjected to 'de novo' peptide sequencing, followed by search for best matching open reading frames inside the $R$. flavefaciens 007 genome contigs arranged in the local database (tBlastn, BioEdit [36]). The translated open reading frames were searched for conserved domains/motifs and annotated using BlastP (www.ncbi.com), Conserved domains, HMMPfam, BlastProDom, HMMSMART, HMMPIR, Superfamily, SignalPHMM/NN, TMHMM, pattern Scan (Prosite), HMMPIR, HMMPanther and HMMSMART (http://www.ebi.ac.uk/Tools/InterProScan/). Signal peptides typical for Gram-positive bacteria were predicted using SignalP 4.0 software (http://www.cbs.dtu.dk/services/SignalP/). Theoretical MW and pIs of all identified $R$. flavefaciens $007 \mathrm{C}$ proteins (without signal sequences) were calculated using Compute pI/MW tool on the Expasy server. Protein sequence similarities were calculated using FASTA sequence alignment software (http:// www.ebi.ac.uk/).

Analysis of type IV Pilus Biogenesis Genes of $R$. flavefaciens $007 \mathrm{C}$

Protein with high N-terminal amino acid similarity to type IV pilins of several bacteria was additionally analyzed using Domain Enhanced Lookup Time Accelerated BLAST (DELTA-BLAST) algorithm and aligned with similar type IV pilins from other bacterial species by Clustal $\Omega$ (www.clustal.org/omega/). The alignment was visualized by Jalview software (www.jalview.org/). The genomic environment of the gene encoding the putative pilin was queried by a combination of ORF Finder and BlastP programs to discover putative genes involved in pili biogenesis. Conserved domains and motifs of the translated products were determined as stated above. Phosphorylation sites in putative type IV pilin were predicted by NetPhosK 1.0 software [37].

\section{Preparation of $R$. flavefaciens Cells for Electron Microscopy}

Cultures of $R$. flavefaciens $007 \mathrm{c}$ and $007 \mathrm{~s}$ were incubated anaerobically as described above with either cellobiose $(18 \mathrm{~h})$ or Avicel cellulose $(48 \mathrm{~h})$. Cells were pelleted by centrifugation at 1 $500 \mathrm{~g}$ for $10 \mathrm{~min}$, fixed with glutaraldehyde and post fixed with osmium tetroxide as described previously [10]. The mounted samples were coated in a thin layer of gold and were observed using a Zeiss EVO MA10 scanning electron microscope.

\section{Supporting Information}

Figure S1 Total cell protein concentrations $\left(\boldsymbol{c}_{\text {prot }}\right)$ and specific avicelase activities of cell (SAA Gel) and supernatant (SAA Sup) fractions during measured during growth of $R$. flavefaciens $007 \mathrm{C}$ and $007 \mathrm{~S}$ on cellobiose, xylan, Avicel and dewaxed cotton. (DOCX)

Figure S2 Major extracellular proteins identified in the cell wall associated (GWAP) fraction of $R$. flavefaciens $007 \mathrm{C}$ and $007 \mathrm{~S}$ grown with cellobiose $(24 \mathrm{~h})$, xylan $(48 \mathrm{~h})$ or Avicel cellulose $(7.5 \mathrm{~d})$ as energy sources. Proteins referred to in Figure 4 are indicated by arrows.

(TIF)

Figure S3 Glustal $\Omega$ alignment of $N$-terminal part of Pil3 (putative type IV pilin of $R$. flavefaciens 007C) with type IV pili from Moraxella bovis, Pseudomonas aeruginosa, Dichelobacter nodosus, Neisseria meningitidis, Myxococcus xanthus, Eikenella corrodens, Neisseria gonorrhoeae in Aeromonas hydrophila.

(DOCX)

Table S1 Major proteins identified in the cell culture supernatant (CCSUP) fraction of $R$. flavefaciens 007G grown on Avicel for 7.5 days.

(PDF)

Table S2 Major extracellular proteins identified in the cell wall associated (GWAP) fraction of $R$. flavefaciens 007C grown on Avicel for 7.5 days.

(PDF)

Table S3 Major extracellular proteins identified in cellulose-bound (GBP) fraction of $R$. flavefaciens $007 \mathrm{C}$ grown on Avicel for 7.5 days.

(PDF)

Table S4 Major extracellular proteins identified in cellulose-bound (GBP) fraction of $R$. flavefaciens $007 \mathrm{C}$ grown on dewaxed cotton for 9.5 days.

(PDF)

Table S5 Major proteins identified in cell culture supernatant (GCSUP) fraction of $R$. flavefaciens 007G grown on dewaxed cotton for 9.5 days.

(PDF)

Table S6 Type IV pili biogenesis cluster of $R$. flavefaciens $007 \mathrm{C}$ and three closest homologues of putative gene products. Abbreviations: $\mathrm{SP}$ - signal peptide, $\mathrm{TMR}(\mathrm{s})$ transmembrane region $(\mathrm{s})$.

(PDF)

\section{Acknowledgments}

We are very grateful to Garry J. Rucklidge for many useful advice and technical assistance in 2-DE protein analysis. We also thank Debbie Wilkinson of the Microscopy and Imaging Core Facility, University of Aberdeen for electron microscopy and Marco Rincon for valuable discussion. E.A.B. holds The Maynard I. and Elaine Wishner Chair of Bio-organic Chemistry. 


\section{Author Contributions}

Conceived and designed the experiments: HJF MV SHD. Performed the experiments: MV MDR LC SHD. Analyzed the data: MV HJF EAB

\section{References}

1. Ding S-Y, Rincon MT, Lamed R, Martin JC, McCrae SI, et al. (2001) Cellulosomal scaffoldin-like proteins from Ruminococcus flavefaciens. J Bacteriol 183: $1945-1953$

2. Rincon MT, Ding S-Y, McCrae SI, Martin JC, Aurilia V, et al. (2003) Novel organization and divergent dockerin specificities in the cellulosome system of Ruminococcus flavefaciens. J. Bacteriol 185: 703-713.

3. Jindou S, Borovok I, Rincon MT, Flint HJ, Antonopoulos DA, et al. (2008) Conservation and divergence in cellulosome architecture between two strains of Ruminococcus flavefaciens. J. Bacteriol 188: 7971-7976.

4. Bayer EA, Lamed R, White BA, Flint HJ (2008) From cellulosomes to cellulosomics. Chem Rec 8: 364-377.

5. Berg Miller ME, Antonopoulos DA, Rincon MT, Band M, Bari A, et al. (2009) Diversity and strain specificity of plant cell wall degrading enzymes revealed by the draft genome of Ruminococcus flavefaciens FD-1. PLoS One 4, e6650. doi: 10.1371/journal.pone.0006650.

6. Rincon MT, Dassa B, Flint HJ, Travis AJ, Jindou S, et al. (2010) Abundance and diversity of dockerin-containing proteins in the fiber-degrading rumen bacterium, Ruminococcus flavefaciens FD-1. PLoS One 5, e12476. doi:10.1371/ journal.pone.0012476.

7. Rincon MT, McCrae SI, Kirby J, Scott KP, Flint HJ (2001) EndB, a multidomain family 44 cellulase from Ruminococcus flavefaciens 17 , binds to cellulose via a novel cellulose-binding module and to another $R$. flavefaciens protein via a dockerin domain. Appl Environ Microbiol 67: 4426-4431.

8. Rincon MT, Cepeljnik T, Martin JC, Barak Y, Lamed R, et al. (2007) A novel cell surface-anchored cellulose-binding protein encoded by the sca gene cluster of Ruminococcus flavefaciens. J Bacteriol 189: 4774-83.

9. Stewart CS, Duncan SH, Flint HJ (1990) The properties of forms of Ruminococcus flavefaciens which differ in their ability to degrade cotton cellulose. FEMS Microbiol Lett 60: 47-50.

10. Stewart CS, Duncan SH, McPherson CA, Richardson AJ, Flint HJ (1990) The implications of the loss and regain of cotton-degrading activity for the degradation of straw by Ruminococcus flavefaciens strain 007. J Appl Microbiol 68: 349-356.

11. Rincon MT, Cepeljnik T, Martin JC, Lamed R, Barak Y, et al. (2005) Unconventional mode of attachment of the Ruminococcus flavefaciens cellulosome to the cell surface. J Bacteriol 187: 7569-7578.

12. Mosoni P, Gaillard-Martinie B (2001) Characterization of a spontaneous adhesion-defective mutant of Ruminococcus albus strain 20. Arch Microbiol 176: $52-61$.

13. Rakotoarivonina H, Jubelin G, Hebraud M, Gaillard-Martinie B, Forano E, et al. (2002). Adhesion to cellulose of the Gram-positive bacterium Ruminococcus albus involves type IV pili. Microbiology 148: 1871-1880.

14. Chamot-Rooke J, Mikaty G, Malosse C, Soyer M, Dumont A, et al. (2011) Posttranslational modification of pili upon cell contact triggers $\mathcal{N}$. meningitidis dissemination. Science 331: 778-782.

15. Dror TW, Morag E, Rolider A, Bayer EA, Lamed R, et al. (2003) Regulation of the cellulosomal CelS (cel48A) gene of Clostridium thermocellum is growth rate dependent. J Bacteriol 185: 3042-3048.

16. Gold ND, Martin VJJ (2007) Global view of the Clostridium thermocellum cellulosome revealed by quantitative proteomic analysis. J Bacteriol 189: 6787-6795.

17. Raman B, Pan G, Hurst GB, Rodriguez M, McKeown GK, et al. (2009) Impact of pretreated switchgrass and biomass carbohydrates on Clostridium thermocellum ATCC 27405 cellulosome composition: a quantitative proteomic analysis. PLoS One 4: e5271.

18. Stevenson DM, Weimer PJ (2005) Expression of 17 genes in Clostridium thermocellum ATCC 27405 during fermentation of cellulose or cellobiose in continuous culture. Appl Environ Microbiol 71: 4672-4678.
BAW. Contributed reagents/materials/analysis tools: KT JP CJY MEBM RL RML. Wrote the paper: MV HJF.

19. Rincon MT, Martin JC, Aurilia V, McCrae SI, Rucklidge GJ, et al. (2004) ScaC, an adaptor protein carrying a novel cohesin that expands the dockerin-binding repertoire of the Ruminococcus flavefaciens 17 cellulosome. J Bacteriol 186: 25762585 .

20. Morisaka H, Matsui K, Tatsukami Y, Kuroda K, Miyake H, et al. (2012) Profile of native cellulosomal proteins of Clostridium cellulovorans adapted to various car bon sources. AMB Express 2: e37.

21. Pegden RS, Larson MA, Grant RJ, Morrison M (1998) Adherence of the Grampositive bacterium Ruminococcus albus to cellulose and identification of a novel form of cellulose-binding protein which belongs to the Pil family of proteins. J Bacteriol 180: 5921-5927.

22. Morrison M, Miron J (2000) Adhesion to cellulose by Ruminococcus albus: a combination of cellulosomes and Pil-proteins? FEMS Microbiol Lett 185: 109115.

23. Rakotoarivonina H, Larson MA, Morrison M, Girardeau J-P, Gaillard-Martinie $\mathrm{B}$, et al. (2005) The Ruminococcus albus pilA1-pilA2 locus: expression and putative role of two adjacent pil genes in pilus formation and bacterial adhesion to cellulose. Microbiology 151: 1291-1299.

24. Jun H-S, GongJ, Qi M, Egbosimba EE, Forsberg CW (2007) Outer membrane proteins of Fibrobacter succinogenes with potential roles in adhesion to cellulose and in cellulose digestion. J Bacteriol 189: 6806-6815.

25. Varga JJ, Nguyen V, O'Brien DK, Rodgers K, Walker R A, et al. (2006) Type IV pili-dependent gliding motility in the Gram-positive pathogen Clostridium perfringens and other Clostridia. Mol Microbiol 62: 680-694.

26. Geissmann TA, Teuber M, Meile L (1999) Transcriptional analysis of the rubrerythrin and superoxide dismutase genes of Clostridium perfringens. J Bacteriol 181: 7136-7139.

27. Sztukowska M, Bugno M, Potempa J, Travis J, Kurtz DM (2002) Role of rubrerythrin in the oxidative stress response of Porphyromonas gingivalis. Mol Microbiol 44: 479-488.

28. Kawasaki S, Ono M, Watamura Y, Sakai Y, Satoh T, et al. (2007) An $\mathrm{O}_{2-}$ inducible rubrerythrin-like protein, rubredoxin, is functional as a $\mathrm{H}_{2} \mathrm{O}_{2}$ reductase in an obligatory anaerobe Clostridium acetobutylicum. FEBS Letts 581: 2460-2464.

29. Weinberg MV, Jenney FE, Cui X, Adams MWW (2004) Rubrerythrin from the hyperthermophilic archaeon Pyrococcus furiosus is a rubredoxin-dependent, ironcontaining peroxidase. J Bacteriol 186: 7888-7895.

30. Kurtz DM (2006) Avoiding high-valent iron intermediates: superoxide reductase and rubrerythrin. J Inorg Biochem 100: 679-693.

31. Hillmann F, Fischer R-J, Bahl H (2006) The rubrerythrin-like protein Hsp21 of Clostridium acetobutylicum is a general stress protein. Arch Microbiol 185: 270-276.

32. Rakotoarivonina H, Terrie C, Chambon C, Forano E, Mosoni P (2009) Proteomic identification of CBM37-containing cellulases produced by the rumen cellulolytic bacterium Ruminococcus albus 20 and their putative involvement in bacterial adhesion to cellulose. Arch Microbiol 191: 379-388.

33. Hungate RE, Stack RJ (1982) Phenylpropanoic acid: growth factor for Ruminococcus albus. Appl Environ Microbiol 44: 79-83.

34. Lever M (1977) Carbohydrate determination with 4-hydroxybenzoic acid hydrazide (PAHBAH): effect of bismuth on the reaction. Anal Biochem 81: 2127.

35. Devillard E, Goodheart DB, Karnati SK, Bayer EA, Lamed R, et al. (2004) Ruminococcus albus 8 mutants defective in cellulose degradation are deficient in two processive endocellulases, Cel48A and Cel9B, both of which possess a novel modular architecture. J Bacteriol 186: 136-145.

36. Hall TA (1999) BioEdit: a user-friendly biological sequence alignment editor and analysis program for Windows 95/98/NT. Nucl Acids Symp Ser 41: 95-98.

37. Blom N, Sicheritz-Pontén T, Gupta R, Gammeltoft S, Brunak S (2004) Prediction of post-translational glycosylation and phosphorylation of proteins from the amino acid sequence. Proteomics 4: 1633-1649. 